\title{
Concurrent instabilities causing multiple rogue waves in infinite-dimensional dynamical systems
}

\author{
Amdad Chowdury • Nail Akhmediev . \\ Wonkeun Chang
}

Received: date / Accepted: date

\begin{abstract}
Complex instabilities are the major reason for drastic changes and extreme events in dynamical systems. Several modes of instability growing simultaneously with nonlinear interaction between them may lead to unforeseeable outcomes leading to catastrophic consequences. The most common examples of these instabilities are the modulation instability (MI). Studies show that an infinite number of instability modes remain active in a dynamical system. Although a one-mode MI can be analyzed in the frame of a precise mathematical model-namely the Akhmediev breather, the dynamics of several concurrent MI modes, referred as the higher-order MI is very difficult to handle. We developed a unique geometrical approach that provides an entirely new and intuitive way to deal with higher-order MI. We apply this approach in description of higher-order modulation instability, multi-breather solutions, their degenerate versions and higher-order rogue waves of the nonlinear Schrödinger equation. For a system with infinitely many interacting instability modes, the band of the instability in this description is a hypercube a multi-dimensional space of modulation frequencies. A large variety of special multi-breather and multi-rogue wave solutions of the nonlinear Schrödinger equation in this description corresponds to special points and lines within this hypercube.
\end{abstract}

Keywords Nonlinear Schrödinger equation · Akhmediev breather · multibreather dynamics

Amdad Chowdury · Wonkeun Chang

School of Electrical and Electronic Engineering,

Nanyang Technological University, Singapore 639798

E-mail: wonkeun.chang@ntu.edu.sg

Nail Akhmediev

Department of Theoretical Physics, Research School of Physics,

The Australian National University, Canberra ACT 2600, Australia 


\section{Introduction}

Instabilities are common in nature [1-4]. They occur in conservative and dissipative systems. In the former case, the system may return back to the point of instability. In the latter case, the system normally moves to the point of stable equilibrium. The complexity of forms of instability is enormous. In simple terms, instability is a growing deviation from the state of equilibrium. Such deviations can have devastating consequences. Examples are atomic reactor catastrophes, tornadoes etc.

Instabilities do exist in both finite-dimensional and infinite-dimensional systems. In the latter case, the degrees of freedom may act collectively in deviating from the state of equilibrium. The most common example of this complex instability is modulation instability (MI) [5-7]. Single frequency MI has been independently studied in many fields of physics. In the case of ocean wave dynamics, it is known as Benjamin-Feir instability, named after the researchers who discovered this phenomenon [8]. In optics, it is known as Bespalov - Talanov instability [9]. The instability leading to spatial skin pattern formation is known as Turing instability $[10,11]$. Instability also leads to washboard structures of unpaved roads [12]. MI can create soliton trains in Bose-Einstein condensates $[13,14]$.

The MI is the growth of a periodic modulation on a continuous wave (CW) background. In MI, the small amplitude modulation initially is exponentially amplified resulting in amplitude deviations comparable to the CW background. In the spectral domain, the MI generates a cascade of spectral sidebands starting with a single pair of them in addition to the central component of the $\mathrm{CW}$ itself.

MI has many practical applications. The MI has been applied to describe the formation of oceanic rogue waves [15-17] and rogue waves in plasma [18]. These ideas have been verified experimentally $[19,20]$. MI can be used for generating a train of ultra-short pulses in optics [21-24], as well as for optical parametric amplification $[25,26]$. The noise-seeded supercontinuum generation from CW pump is driven by MI [27-29]. The emergence of optical rogue waves in chaotic wave fields is a direct consequence of MI [30,31].

In the early days, investigations on MI have been limited to the linear stability analysis of constant amplitude waves $[8,9,32]$. It was later realised that a long-time evolution of MI can be modelled by the nonlinear Schrödinger equation (NLSE) $[33,34]$. While the analytical solution of NLSE, called the Akhmediev Breather (AB) that describes full growth-return cycle of MI, has existed for more than 30 years $[35,22,36]$, most theoretical investigations, until recently, have been carried out using numerical approaches and finite spectrum approximations [37]. Only in the past ten years, researchers have come to realise that the $\mathrm{AB}$ is a versatile tool that can be applied to study complex dynamics of MI including the nonlinear stage of its evolution [38], multi-frequency initial conditions [39], in media with variable parameters [40] and for studies of Fermi-Pasta-Ulam recurrence [41-43]. 
A single modulation frequency that grows on a CW background leads to the development of a fundamental AB solution. This is an idealised case. Noise, being the major generator of MI, consists of many frequency components. Each component leads to the growth of an individual $\mathrm{AB}$ connected to that frequency. This leads to a highly complex evolution due to the interactions among multiple ABs $[22,36]$. One such example is the initial stage of the noise-seeded supercontinuum generation process [27]. These interactions can be described using multi-breather exact solutions of the NLSE that can be derived using Darboux transformation scheme [36]. These breather solutions generally pose significant challenges, because their theoretical descriptions entail highly complex mathematical formalisms. Moreover, the mathematical complexity increases with the rising number of instability modes. As these solutions are highly involved, it is worthy to develop other approaches that might simplify their understanding.

In this work, we introduce a general description of the multi-AB solutions in $N$-dimensional Euclidean geometrical space. By applying the geometrical interpretations of MI that we introduce here, one can almost entirely sidestep the cumbersome mathematics, while still accurately pinpoint the precise characteristics of the MI and the multi-AB solution dynamics. We provide a complete account of NLSE multi-breather dynamics by transforming the rigorous mathematical interpretation into a more simplified and intuitive geometrical configuration. This new technique will allow us to understand the complex nature of MI in chaotic wave fields and extreme nonlinear wave localisation in various systems such as hydrodynamics, nonlinear optics, plasma and BoseEinstein condensates.

\section{First-order $\mathrm{MI}$ and $\mathrm{AB}$ solution}

In its simplest form, the NLSE is given by:

$$
i \psi_{x}+\frac{1}{2} \psi_{t t}+|\psi|^{2} \psi=0
$$

where $x$ is the propagation and $t$ is the transverse variable. $\psi(x, t)$ is the complex envelope of the wave field. Eq. (1) is integrable and permits analytical solutions including solitons, breathers, rogue waves and double periodic solutions, i.e. solutions periodic both in $x$ and $t$ : [44-49]. The breathers are a class of solutions that represent the nonlinear stage of evolution that starts from a plane wave distorted with a small periodic perturbation. They can be derived either using special ansatz [35] or using the Darboux transformation technique [36]. The one parameter family of breather solutions can be written in the form [44]:

$$
\psi_{1}=e^{i x}\left(-1+\frac{\kappa\left\{\kappa^{2} \cosh (x \delta)+2 i \delta \sinh (x \delta)\right\}}{2\{\kappa \cosh (x \delta)-\delta \cos (t \kappa)\}}\right)
$$


where $\kappa$ is the initial modulation frequency and $\delta=\frac{\kappa}{2} \sqrt{4-\kappa^{2}}$ is the growth rate of the modulation that is determined by the initial modulation frequency. These parameters are the same as for the MI that starts the breather. Therefore, $\kappa$ here is the only free parameter that fully characterizes the fundamental breather solution dynamics such as its periodicity, growth rate, and maximal amplitude. The AB solution (2) can be generalised by adding two more free parameters, such as variable amplitude and velocity through the scaling and Galilean transformations [44]. However, these do not influence general concepts presented in this work.

The growth rate of MI against the modulation frequency is plotted in Fig. 1(a) for both positive and negative frequencies. This plot shows that only the spectral components with frequencies within the instability band, $-2<\kappa<2$, will be amplified. Breathers exist at any point of this interval (Line $\mathrm{B}^{\prime} \mathrm{B}$ ) including $\kappa=0$. The latter case corresponds to the Peregrine solution [50] also known as a fundamental rogue wave [51,52]. It is the limiting case of the solution (2) when $\kappa \rightarrow 0$. As the growth rate is symmetrical around the carrier frequency $(\kappa=0)$, we can restrict ourselves to only positive $\kappa$, which is the line $\mathrm{AB}$, in our plot. Thus, only $\kappa \geq 0$ will be considered in all subsequent analysis.
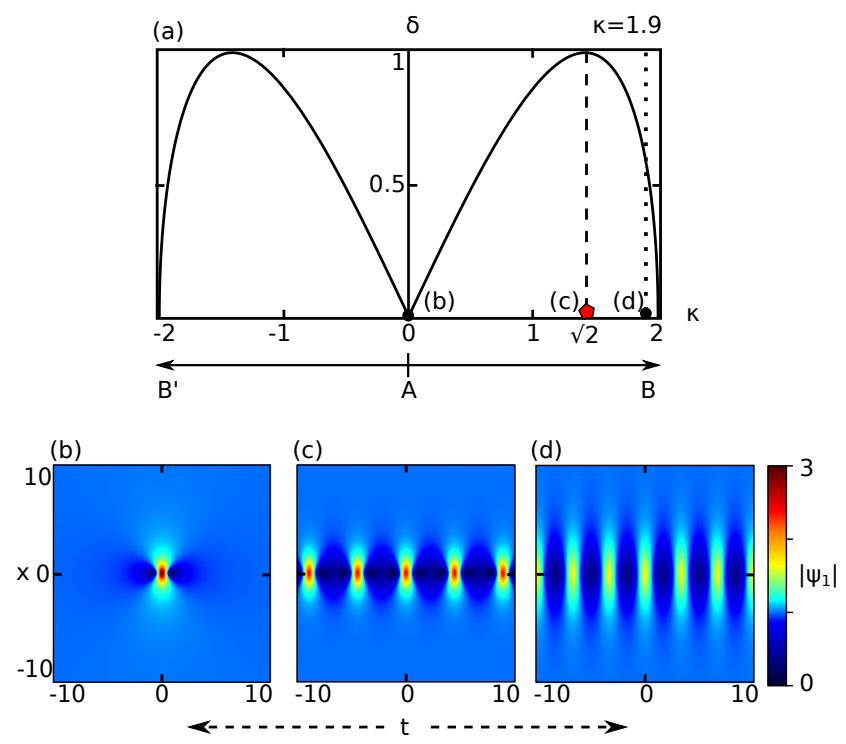

Fig. 1 (Color online) (a) Instability band for the MI with frequency $\kappa$ which is the region of existence for the first-order breather solution Eq. (2). Panels (b)-(d) show false colour amplitude plots of the solution: (b) Fundamental rogue wave solution when $\kappa \rightarrow 0$ at the point $A$ in (a). (c) Breather solution at the point of the highest gain at $\kappa=\sqrt{2}$ of the instability band marked by the red diamond in (a). (d) Breather solution at $\kappa=1.9$ marked by the black solid dot in (a). 
The breather has its largest growth rate $\delta=1$ at $\kappa=\sqrt{2}$ as indicated in Fig. 1(a) with a red diamond. The false colour amplitude plot of the corresponding breather solution is illustrated in Fig. 1(c). As $\kappa \rightarrow 2$, the growth rate of the breather reduces to zero and its amplitude becomes smaller. An example is shown in Fig. 1(d) for $\kappa=1.9$. This breather clearly has a much lower peak amplitude and the growth rate. On the contrary, when $\kappa \rightarrow 0$, the limit is the rogue wave with a single peak of the highest amplitude 3 as illustrated in Fig. 1(b). This is a singular event in time and space [53].

Thus, as we move along the line from A to B in Fig. 1(a), the peak amplitude of the breather decreases while the number of pulses per unit $t$ in the pulse train increases. On the other hand, the growth rate, shown in Fig. 1(a), increases until the maximum is reached at $\kappa=\sqrt{2}$, and falls again down to zero beyond this point. All amplitude plots in Fig. 1(b)-(d) are shown with the middle peak centred at the origin. Translations along the $x$ and $t$ axes are possible but will be ignored here. This is the special case that we limit our attention to in the present work. This is also the case for multi-breather plots presented below. Their description requires higher-dimensional Euclidean frequency space as shown in the subsequent sections.

\section{Second-order MI and two-breather solutions}

MI may have two or more side-bands growing simultaneously within the instability band. This case can be accurately modelled by the two-breather solution [22] and its higher-order extensions [36]. As the frequencies of the two sidebands are independent parameters, their influence on the 2 - breather solution must be represented on a plane map rather than on a single line AB. Condition for simultaneous existence of the two breathers is that both pairs of sidebands must be within the instability band. Their frequencies and initial amplitudes are independent parameters. As said before, we choose the initial amplitudes such that the two breathers are centred at $x=0$. Then only the two frequencies remain independent. In this case, the two-breather solution will be the nonlinear superposition of two first-order breather solutions Eq. (2) with maximal interaction. The exact solution of this type can be found in [22] and the way of deriving it in [36]. For the two-breather solution, there are two MI frequency parameters $\kappa_{1}$ and $\kappa_{2}$ which separately provide the associated growth rates, $\delta_{1}=\frac{1}{2} \kappa_{1} \sqrt{4-\kappa_{1}^{2}}$ and $\delta_{2}=\frac{1}{2} \kappa_{2} \sqrt{4-\kappa_{2}^{2}}$. These two frequencies are plotted along the horizontal and vertical axes in Fig. 2. The two breathers are of similar size in $x$-direction when their growth rates coincide. Naturally, this happens when $\kappa_{1}=\kappa_{2}$. Alternatively, this also happens when $\kappa_{2}= \pm \sqrt{4-\kappa_{1}^{2}}$. The lines of equal growth rate $\delta_{1}=\delta_{2}$ are shown in Fig. 2. These are the diagonal line $\mathrm{CF}$ and the circular arc $\mathrm{EQE}^{\prime}$. Solutions on the diagonal line where $\kappa_{1}=\kappa_{2}$ are degenerate. As such, they require special technique for derivation [54].

A smooth transition from a two-breather solution to a second-order or a first-order rogue waves happens only on the lines of equal growth-rate. The 


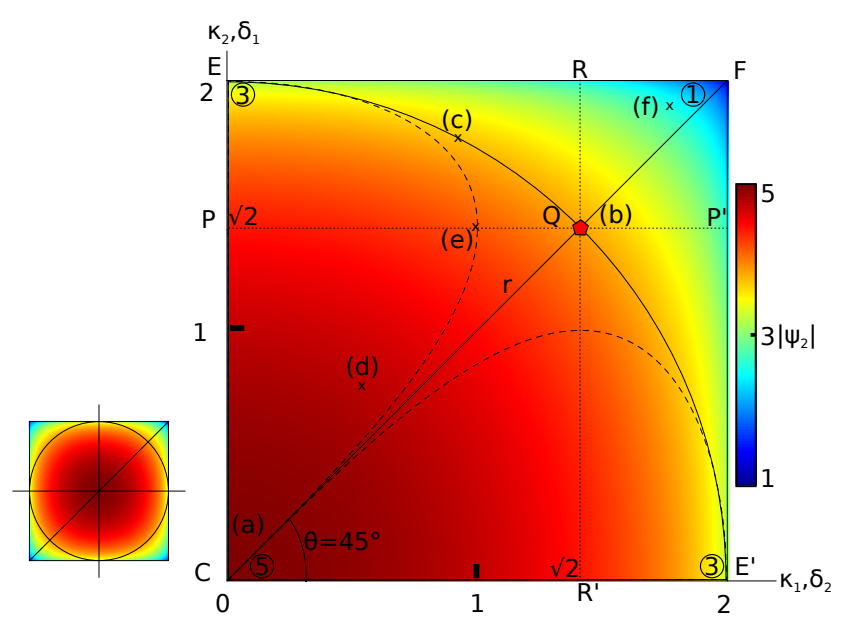

Fig. 2 (Color online) The square of instability for the two-breather solution on the plane of two frequencies $\kappa_{1}$ and $\kappa_{2}$. The colour scale shows the maximal amplitude of the solution. The diagonal $\kappa_{2}=\kappa_{1}(\mathrm{CF})$ and the circle $\kappa_{2}= \pm \sqrt{4-\kappa_{1}^{2}}\left(\mathrm{EQE}^{\prime}\right)$ are the lines of equal growth rates. They intersect at the point $\mathrm{Q}$, where $\kappa=\sqrt{2}$. The two black dashed curves are the growth rates of each individual breather while the two black dotted lines show their maximum growth rates. The two growth rates are both maximal at the point $\mathrm{Q}$. The maximal amplitude within the circle $\mathrm{EQE}^{\prime}$ is higher than outside of it. The inset shows the complete region of instability for poshtive and negative frequencies.

point $\mathrm{C}$ is the one where the two breather solution is transformed into secondorder rogue wave [51]. This happens when we move along the diagonal line CF to the point $\mathrm{C}$. This is the point of the highest amplitude 5 of the two-breather solution. Similarly, the growth rates are equal on the circle EQE'. The two breather solution is transformed into a first-order rogue wave at each corner point $\mathrm{E}$ and $\mathrm{E}^{\prime}$ shown in Fig. 2. This happens because one of the breathers (with $\kappa \rightarrow 0$ ) is transformed into the first-order rogue wave while the other one (with $\kappa \rightarrow 2$ ) reduces its amplitude and merges with the background. As said, the point $\mathrm{C}$ in Fig. 2 corresponds to the second order rogue wave. Its amplitude profile is shown in Fig. 3(a). The periods of the two breathers at this point are infinite. A slight deviation from this point leads to finite periods of the two breather components.

The plane of two frequencies $\left(\kappa_{1}, \kappa_{2}\right)$ can also be described by the polar coordinates $(r, \theta)$. The radius $r$ in these co-ordinates influences the amplitudes and the growth rates of the two breather components. The angle $\theta$ determines their frequency ratios. The maximal amplitude at the centre of the two-breather solution is given by the sum of the background and individual amplitudes of the two components:

$$
\psi_{2}(x=0, t=0)=1+\sqrt{4-\kappa_{1}^{2}}+\sqrt{4-\kappa_{2}^{2}}
$$

The linear addition follows from the exact two-breather solution [36]. It does not mean that this solution is a linear superposition. This maximal amplitude 


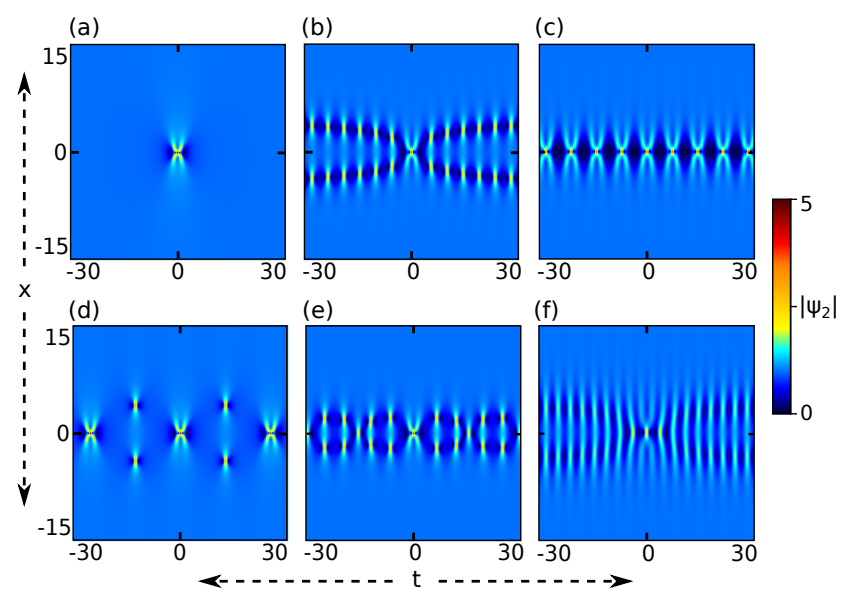

Fig. 3 (Color online) False-colour plots of the two-breather solution at various points on the 2D instability plane in Fig. 2. Panel (a) shows the second-order rogue wave at point $\mathrm{C}$ $\left(\kappa_{1}, \kappa_{2}\right)=(0,0)$. Panel (b) shows the degenerate two-breather solution at the point $\mathrm{Q}$ (reddiamond) where $\left(\kappa_{1}, \kappa_{2}\right)=(\sqrt{2}, \sqrt{2})$. Panel (c) corresponds to either $\left(\kappa_{1}, \kappa_{2}\right)=(0.89,1.78)$ or $(1.78,0.89)$. Panel $(\mathrm{d})$ is at $(0.5,0.75)$. Panel $(\mathrm{e})$ is at $(1.0, \sqrt{2})$ and panel $(\mathrm{f})$ is at $(1.8,1.9)$.

is shown, in Fig. 2, in colour scale. It is highest $(=5)$ at the point $\mathrm{C}$ and lowest $(=1)$ at the point $\mathrm{F}$ along the diagonal line. As the solution along this line is degenerate $\left(\kappa_{1}=\kappa_{2}\right)$, it resembles collision of two breathers. This solution is shown in Fig. 3(b) for the case of maximal growth rate at the point marked by red diamond in Fig. 2. Closer to the point F, the amplitude of the two breather solution reduces to the level of the background. The plot of the solution near this point is shown in Fig. 3(f).

The growth rates of the two breathers within the solution remain the same as for each of them in absence of interaction. The growth rates are zero at the points $\mathrm{C}$ and $\mathrm{F}$. Only one of them is zero at the sides of the square, i.e. on the lines $\mathrm{EF}$ and $\mathrm{FE}^{\prime}$. The solution at these lines reduces to a single breather.

The common growth rate of the degenerate breather excited on the line CF increases from 0 to a maximum value the point $\left(2,45^{\circ}\right)$ (the red diamond) and reduces to 0 again at the point $\mathrm{F}$. The points within the area $\mathrm{CQP}$ are at the rising part of the growth rate curve. The area QFR corresponds to the falling part of the growth rate curve. However, the points within the rectangular area PQRE have both the rising and falling parts of the growth rate curve. One of the two breathers excited in this area has always higher growth rate than the other one except for the circular arc QE.

The radial distance $r$ controls both the amplitude variation and the periodicity of the breathers $T=2 \pi / \kappa_{j}$. The increase of $r$ leads to reduction of the amplitude and the periodicity of the individual breathers. When $r$ increases, breathers are transformed into low amplitude, densely peaked periodic pulse trains. In the limit $\kappa_{j} \rightarrow 2$, they are collapsed into the background. On the other hand, in the limit $\kappa_{j} \rightarrow 0$, the period of the two breathers becomes 
infinite and the solution turns into a second order rogue wave with the highest amplitude 5 as shown in Fig. 3(a).

Angular deviations from the diagonal line CF in Fig. $2\left(\theta \rightarrow 45^{\circ}+\Delta \theta\right)$ lead to frequency ratios different from 1 . On the line $\mathrm{CF}$, which is degenerate case, breathers have one collision point at the origin. This case is illustrated in Fig. 3(b). Deviation from this line leads to periodic collisions defined by the beating frequency $\Delta \kappa=\kappa_{1}-\kappa_{2}$ that, in turn, depends on the angular deviation $\theta$. If the ratio of MI frequencies $\kappa_{1}: \kappa_{2}$ is a ratio of two rational numbers, i.e. if the frequencies are commensurate, the periodically located points of collisions have the peaks with the same amplitude as at the origin. This can be seen in Fig. 3(c), calculated for the case $\kappa_{1}: \kappa_{2}=1: 2$, where the superposition creates a regular breather collision pattern. Another example is shown in Fig. 3(d) where the $\kappa_{1}: \kappa_{2}=2: 3$. However, if the ratio $\kappa_{1}: \kappa_{2}$ is an irrational number, the pattern is not periodic. One example is shown in Fig. $3(\mathrm{e})$ where $\kappa_{1}: \kappa_{2}=1: \sqrt{2}$.

Closer to the vertical line $\mathrm{CE}$ in Fig. 2, the ratio $\kappa_{1}: \kappa_{2} \rightarrow 0$. The combined breather amplitude here also decreases. At the point E, $\kappa_{1} \rightarrow 0$. Only one central peak remains at the origin with the amplitude $\psi(0,0)=3$ as it follows from Eq. (3). This solution is the Peregrine breather. Due to the symmetry, the point $\mathrm{E}^{\prime}$ also corresponds to the Peregrine breather. On the circle $r=2$, connecting the points $\mathrm{E}$ and $\mathrm{E}^{\prime}$, the growth rates of the two breather components are equal.

\section{Third-order MI and three-breather solution}

When three pairs of side-bands are involved in the modulation instability, the evolution may lead to a 3 -breather solution. The three frequencies $\kappa_{j}$ $(j=1,2,3)$ must be independent but located within the instability band. They are the defining parameters for the third-order breather solution. The corresponding growth rates are $\delta_{j}=\frac{\kappa_{j}}{2} \sqrt{4-\kappa_{j}^{2}}$.

The features of the three-breather solution can be described using a threedimensional plot instead of a plane like in Fig. 3. A cube replaces the square and a sphere replaces the circle as shown in Fig. 4(a). As before, it is sufficient to deal with only positive values of frequencies. Then, we can restrict ourselves with 1/8-th of this cube. It is shown in Figs. 4(b) and 4(c) in two different orientations.

The three MI frequencies can be represented in spherical co-ordinates $(x, y, z) \rightarrow(r, \theta, \phi)$ with $\theta$ and $\phi$ being the polar and the azimuthal angles as shown in Fig. 4(d). In this parameter space, $\kappa_{1}$ and $\kappa_{2}$ vary along the $x$ and $y$ axes respectively, while $\kappa_{3}$ varies along the $z$ axes. The growth rates are equal on a surface given by $\delta_{1}=\delta_{2}=\delta_{3}$. Solving these equations gives us 5 sets of real solutions. Each set contains the values of $\kappa_{1}$ and $\kappa_{2}$ expressed in 
(a)

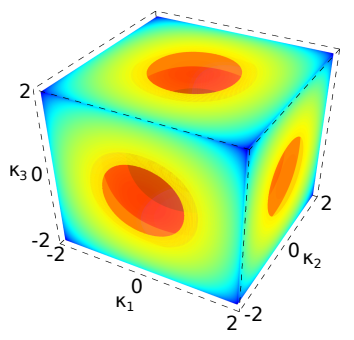

(b)
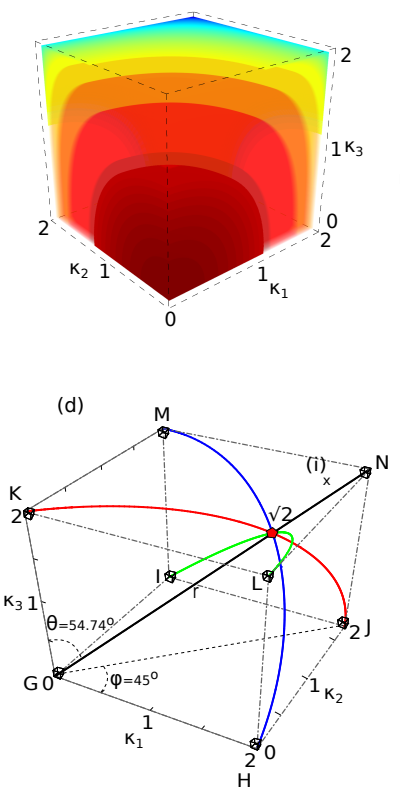

(c)

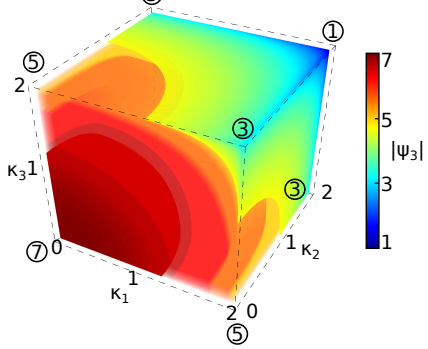

Fig. 4 (Color online) The cube of instability in the 3D space of modulation frequencies $\left(\kappa_{1}, \kappa_{2}, \kappa_{3}\right)$. (a) The full cube that includes positive and negative frequencies. (b) part of the same cube with only positive frequencies. (c) Same as in (b) from different perspective. Encircled numbers at the vertices show the maximal amplitude of the composite breather. Colour coding in (a), (b) and (c) shows the maximal amplitude of the complex breather solution. (d) The same cube as in (b) with coloured curves that correspond to the lines of equal growth rates of breather components.

terms of $\kappa_{3}$ :

$$
\left[\begin{array}{c}
\left(\kappa_{3}, \kappa_{3}\right),\left( \pm \sqrt{4-\kappa_{3}^{2}}, \kappa_{3}\right) \\
\left(\kappa_{3}, \pm \sqrt{4-\kappa_{3}^{2}}\right),\left( \pm \sqrt{4-\kappa_{3}^{2}}, \pm \sqrt{4-\kappa_{3}^{2}}\right) \\
\left( \pm \sqrt{4-\kappa_{3}^{2}}, \mp \sqrt{4-\kappa_{3}^{2}}\right)
\end{array}\right]
$$

These solutions are the combination of two circles $\kappa_{1}^{2}+\kappa_{3}^{2}=4$ and $\kappa_{2}^{2}+\kappa_{3}^{2}=4$. They are shown in Fig. 4(d). The second and third solutions are shown by the blue and green circular orbits respectively, the fourth solution is shown by the red circle in Fig. 4(d). The third circle appears when the first two circles vary along the common axes $\kappa_{3}$. Solutions with negative $\kappa_{i}$ are excluded. These three circles in the $3 D$ parameter space define a sphere $\kappa_{1}^{2}+\kappa_{2}^{2}+\kappa_{3}^{2}=4$, centered at the origin. The central diagonal axis is shown by the solid-black line GN in Fig. 4(d). The three-breather solution on this line is degenerate. The central axis and the circular curves mentioned above are located within the cube $0<\kappa_{j}<2(j=1,2,3)$. A complete description of the dynamics of a three-breather solution can be done using this cube and the sphere within it. 
The maximal amplitude of the solution $\psi_{3}(0,0)$ is given by:

$$
\psi_{3}(x=0, t=0)=1+\sqrt{4-\kappa_{1}^{2}}+\sqrt{4-\kappa_{2}^{2}}+\sqrt{4-\kappa_{3}^{2}}
$$
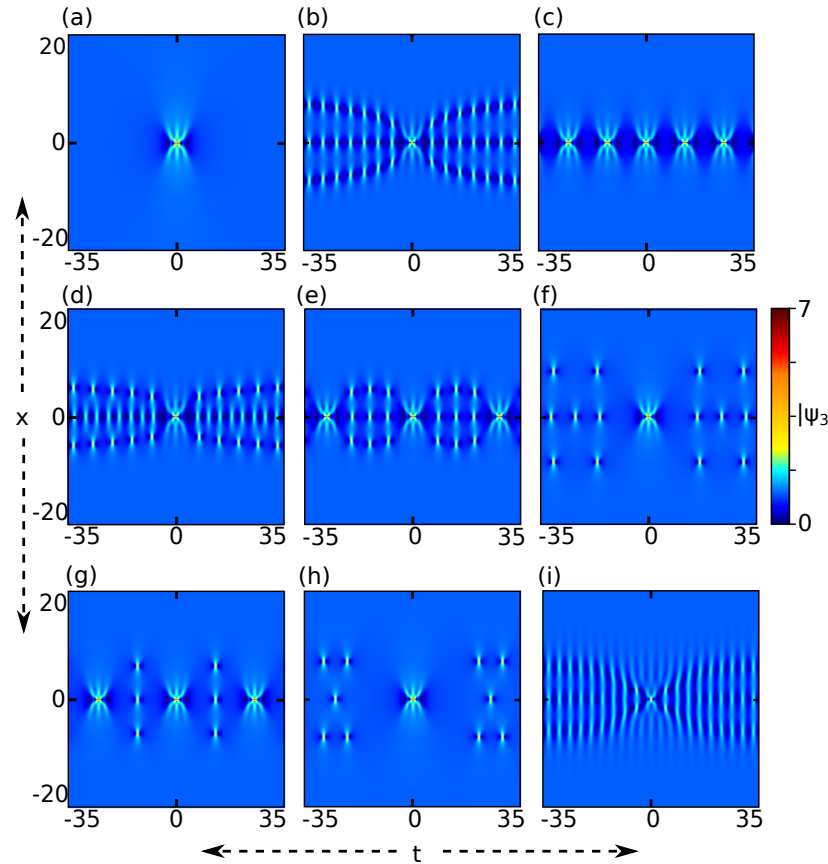

Fig. 5 (Color online) A third-order NLSE breather solution for the values of frequencies: (a) $\left(\kappa_{1}, \kappa_{2}, \kappa_{3}\right)=(0,0,0)$, (point G in Fig. 4) (b) $(\sqrt{2}, \sqrt{2}, \sqrt{2})$, (red-diamond in Fig. 4), (c) $(1,0.5,1.5)$, (d) $(1, \sqrt{3}, 1)$, (a point located on the green circular curve in Fig. 4), (e) $(0.9,1.35,1.125)$, (f) $(0.8,0.4,0.534),(\mathrm{g})(1,0.5,0.75)$, (h) $(0.5,0.25,0.625),(\mathrm{i})(1.7,1.8,1.9)$, (the point is located near the end of the diagonal line in Fig. 4).

On the surface of the sphere, the growth rates remain equal on the three circular curves shown in Fig. 4(d). The growth rates decrease beyond the spherical surface. Given the symmetry of the cube, we are able to consider only one part of it. At the centre of the sphere (point $\mathrm{G}$ ), all the three MI frequency components are zeros $\left(\kappa_{1}, \kappa_{2}, \kappa_{3}\right)=(0,0,0)$. Here, the solution is converted to the third-order rogue wave. It is illustrated in color-coded amplitude plot in Fig. 5(a). On the diagonal line $G N$, the solution is degenerate. Here, $\kappa_{1}: \kappa_{2}$ : $\kappa_{3}=1: 1: 1$. The amplitude along this line decreases from $\psi_{3}(0,0)=7$ at the point $\mathrm{G}$ to $1+3 \sqrt{2}$ at the point $(r, \theta, \phi)=\left(\sqrt{6}, 54.74^{\circ}, 45^{\circ}\right)$ (shown by the red diamond). Simultaneously, the growth rate increases from 0 to 1 . The maximal amplitude is presented in two different view angles in Figs. 4(b) and 4(c) in a colour scale. An example of the degenerate three breather solution at the point of its highest gain is illustrated in Fig. 5(b). The maximal amplitude decreases 
from the point of maximal growth rate from $1+3 \sqrt{2}$ to 1 at the point $\mathrm{N}$ where the solution is converged to the continuous wave background. The growth rate simultaneously decreases from 1 to 0 . Small deviations from the degenerate case keep similar structure. The solution at the point close to $\mathrm{N}$ that has the frequency components $\left(\kappa_{1}, \kappa_{2}, \kappa_{3}\right)=(1.7,1.8,1.9)$ is illustrated in Fig. 5(i). The amplitudes of this solution are nearly at the level of the background.

When the breather frequencies are commensurate, the composite breather solution is periodic. For example, Fig. $5(\mathrm{c})$ shows the case of $\kappa_{1}: \kappa_{2}: \kappa_{3}=1$ : $2: 3$. This solution is clearly periodic. Solutions in Figs. $5(\mathrm{e}),(\mathrm{g})$ and $(\mathrm{h})$ are also periodic as the three frequencies here are also commensurate.

Two of the frequencies $\kappa$ are equal to 2 at the end points of the circular curves in Fig. 4(d). Solution at these points turns into lower-order rogue waves. One of these points is the vertex $\mathrm{L}$ in Fig. 4(d). It has cartesian co-ordinates $(2,0,2)$ or polar co-ordinates $(r, \theta, \phi)=\left(2 \sqrt{2}, 45^{\circ}, 0\right)$. As the two frequency components $\kappa_{1}=\kappa_{3} \rightarrow 2$, the breather components corresponding to these values disappear into the background. The frequency $\kappa_{1} \rightarrow 0$ corresponds to the first-order rogue wave. Thus, the three-breather solution converges to the fundamental rogue wave. Its maximal amplitude is 3 as shown in Fig. 4(c). On the contrary, the vertices I,H and K in Fig. 4(d) correspond to the secondorder rogue waves as two of the frequencies are zeros while the third one is 2 . Their maximal amplitudes are 5 as shown in Fig. 4(c).

\section{$5 N$-order $\mathrm{MI}$ and $N$-breather solutions}

The number of individual breathers in the composite solution can be increased to infinity [36]. Then the solution can be analysed for arbitrary value of $N$. The space for unstable frequencies of an $N$-breather solution forms an $N$ dimensional hypercube. Each of these dimensions corresponds to a pair of MI frequency side-bands $\kappa_{j}$ with $j=1,2,3 \cdots N$ located within the instability band. Instabilities will lead to the development of an $N$-breather solution with equal number of individual growth rates $\delta_{j}=\frac{\kappa_{j}}{2} \sqrt{4-\kappa_{j}^{2}}$. Similar to the cases of 2 and 3 dimensions, we can calculate the curves of equal growth rates $\delta_{1}=\delta_{2}=\delta_{3}=\cdots \delta_{N}$. Considering only the positive real frequencies, we can find these curves from:

$$
\sum_{j=1}^{N} \kappa_{j}^{2}=\kappa_{1}^{2}+\kappa_{2}^{2}+\kappa_{3}^{2}+\cdots+\kappa_{N}^{2}=4
$$

The volume $\left(V_{N}\right)$ and the surface $\left(S_{N}\right)$ area of an $N$ - sphere with the radius 2 is given as [55]:

$$
V_{N}=\frac{2^{N} \pi^{N / 2}}{\Gamma\left(\frac{N}{2}+1\right)}, \quad S_{N}=\frac{2^{N} \pi^{N / 2}}{\Gamma\left(\frac{N}{2}\right)}
$$

where $\Gamma$ is a gamma function. Within this $N$ - sphere, the individual growth rates of an $N$-breather solution increase with the radius $r$ until reaching the 
surface of the hypersphere. The growth rates decay outside of it. The total number of curves of equal growth rates on the surface of the $N$ - sphere is $\frac{1}{2}\left(2^{N}-2\right)$.

A hypercube has $2^{N}$ vertices and for an $N$-breather solution, each of these vertices is the end point of these curves. The composite breather solution is transformed into a lower-order rogue waves at these points. The centre point of the $N$ - sphere where all frequencies are zero is the location of the highest $N^{t h}$-order rogue wave. The maximal amplitude of the $N$-breather solution at $\psi_{N}(x=0, t=0)$ is given by:

$$
\psi_{N}(x=0, t=0)=1+\sum_{j=1}^{N} \sqrt{4-\kappa_{j}^{2}}
$$

Despite the composite solution is nonlinear, the maximal amplitude is the linear sum of the individual breather amplitudes.

When more than three MI modes grow simultaneously, it is seemingly difficult to draw and conceive the plot of instability geometrically. However, the hypercube is a regular geometrical object that can be presented as a twodimensional graph [57]. An example of this construction for the 5-dimensional case is shown in Fig.6. The vertices and edges of the $N$-dimensional hypercube are shown explicitly. The hypercube has $2^{N-1} N$ edges. Each edge represents one MI mode with variable frequency $\kappa_{j}$, the vertices are the locations of the rogue waves. The central vertex at the origin corresponds to the $N$-th order rogue wave. The vertex located at the opposite end of the hypercube (behind the orange point in Fig.6) corresponds to the plane wave. The main principles of this construction are clear from Fig.6.

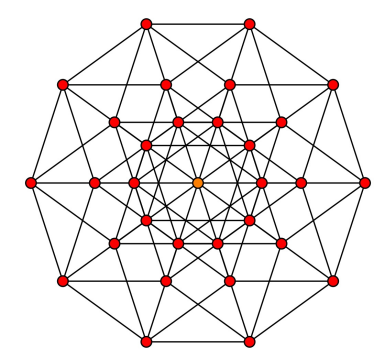

Fig. 6 (Color online) The vertices and edges of the hypercube of instability in the 5-D space. The central vertex (orange) corresponds to the 5 -th order rogue wave. The circle of vertices with the smallest radius corresponds to the 4 -th order rogue waves. The next layer of vertices corresponds to the 3-rd order rogue waves etc. 


\section{Conclusions}

In many problems of interest and, generally, in nature, instability occurs in a continuous range of initial frequencies. Thus, it is not surprising that several modes of instability may start simultaneously and develop concomitantly. The results can be devestating when we deal with the ocean waves or other natural phenomena. Understanding these phenomena is important for modeling them and predicting the consequences when possible. Single-mode instability in most of the cases can be described analytically using exact solutions or direct numerical modeling of the problem. However, multi-mode instability is another matter. Each mode, once excited remains in the system. Describing these phenomena in the space of several parameters is a challenging task.

Using geometrical interpretation, we provide new intuitive approach to deal with multi-mode instability bypassing the immensely complex mathematical formalism. We provided one example of qualitative analysis of this type of problem that can be directly applied to the ocean wave dynamics or nonlinear fiber optics. First step in experimental observation of such instabilities have been done in [6] and [56]. With some modifications, our technique can be extended to other cases of multi-mode instabilities that lead to catastrophic events such as tornados, oceanic rogue waves and various other cataclysmic events in earth weather system that cause losses of human lives and overwhelming damage to ships and structures on land. Multi-mode instabilities also lead to stock market crashes, communication system failulres, biological system anomalies and human stampedes. Thus, the ways they are treated is vitally important for applications. Qualitative analysis suggested in our work may lead to further progress in this area of research.

\section{Conflict of interest}

The authors declare that they have no conflict of interest.

\section{References}

1. Kawamura, K. et al.: State dependence of climatic instability over the past 720,000 years from Antarctic ice cores and climate modeling. Sci. Adv. 3, e1600446 (2017)

2. Alipour M.J., Lashkari A.: Sand instability under constant shear drained stress path. Int. J. of Solids and Structures, 150, 66-82 (2018)

3. Lashmore-Davies C.N., McCarthy D.R., and Thyagaraja A.: The nonlinear dynamics of the modulational instability of drift waves and the associated zonal flows. Phys. of Plasmas 8 p. 5121 (2001)

4. Samuelson P.A.: Scaling and dynamics of washboard roads. Econometrica 10, 1-25 (1942)

5. Wabnitz S., and Akhmediev N.: Efficient modulation frequency doubling by induced modulation instability., Optics Communications, 283, 1152 (2010)

6. Erkintalo M., Hammani K., Kibler B., Finot C., Akhmediev N., Dudley J.M., and Genty G.: Higher-Order Modulation Instability in Nonlinear Fiber Optics. Phys. Rev. Lett. 107, 253901 (2011) 
7. Guo, D. and Tian, S.-F. and Zhang, T.-T. and Li, J.: Modulation instability analysis and soliton solutions of an integrable coupled nonlinear Schrödinger system, Nonlinear Dyn. 94, 2749-2761, (2018)

8. Benjamin T.B.: Instability of periodic wavetrains in nonlinear dispersive systems. Proc. R. Soc. Lond. A. 299, 59-76 (1967)

9. Bespalov V.I., Talanov V.I.: Filamentary structure of light beams in nonlinear liquids. ZhETF Pisma Redaktsiiu, 3, 471 (1966)

10. Turing A.M.: The chemical basis of morphogenesis. Philos. Trans. of the Royal Soc. of Lon. Series B, Biological Sciences, 237, 37-72 (1952)

11. Zheng Q. and Jianwei S.: Turing instability in a gene network with cross-diffusion. Nonlinear Dyn. 78, 1301-1310 (2014)

12. Bitbol A.F., Taberlet N., Morris S.W., McElwaine J.N.: Scaling and dynamics of washboard roads. Phys. Rev. E. 79, 061308 (2009)

13. Nguen J.H.V., Luo D., Hulet R.G.: Formation of matter-wave soliton trains by modulational instability. Sci. 356, 422-426 (2017)

14. Song N., Zhang W. and Yao M.h.: Complex nonlinearities of rogue waves in generalized inhomogeneous higher-order nonlinear Schrödinger equation. Nonlinear Dyn. 82, 489-500 (2015)

15. Garrett C., Gemmrich J., Rogue waves. Phys. Today, 62, 62-63 (2009)

16. Onorato, M., Residori, S., Bortolozzo, U., Montina, A., Arecchi, F.T.: Rogue waves and their generating mechanisms in different physical contexts. Phys. Rep. 528, 47-89 (2013)

17. Chen, S., Baronio, F., Soto-Crespo, J.M., Grelu, P., Mihalache, D.: Versatile rogue waves in scalar, vector, and multidimensional nonlinear systems. J. Phys. A Math. Theor. 50, 463001 (2017)

18. Guo S., Mei L., He Y., Li Y.: Modulation instability and ion-acoustic rogue waves in a strongly coupled collisional plasma with nonthermal nonextensive electrons. Plasma Phys. Control. Fusion, 58, 025014 (2016)

19. Chabchoub A., Hoffmann N.P., Akhmediev N.: Rogue wave observation in a water wave tank. Phys. Rev. Lette. 106, 204502 (2011)

20. Tsai Y.Y., Tsai J.Y., Lin I.: Generation of acoustic rogue waves in dusty plasmas through three-dimensional particle focusing by distorted waveforms. Nat. Phys., 12, 573-577 (2016)

21. Hasegawa A., Brinkman W.F.: Tunable coherent ir and fir sources utilizing modulational instability. IEEE J. Q. Electron. 16, 694-697 (1980)

22. Akhmediev N., Eleonsky V.E., Kulagin N.: Generation of periodic trains of picosecond pulses in an optical fiber: exact solutions. Sov. Phys. JETP, 62, 894-899 (1985)

23. Akhmediev, N., Dudley, J.M., Solli, D.R., Turitsyn, S.K.: Recent progress in investigating optical rogue waves. J. Opt. 15, 060201 (2013)

24. Malomed, B.A., Mihalache, D.: Nonlinear waves in optical and matter-wave media: A topical survey of recent theoretical and experimental results. Rom. J. Phys. 64, 106 (2019)

25. Greer E.J, Patrick D.M, Wigley P.G.J, Taylor J.R.: Generation of 2 THz repetition rate pulse trains through induced modulational instability. Electron. Lett. 25, 1246-1248 (1989)

26. Agrawal G.P.: Modulation instability induced by cross-phase modulation. Phys. Rev. Lett. 59, 880 (1987)

27. Dudley J.M., Genty G., Dias F., Kibler B., Akhmediev N.: Modulation instability, Akhmediev Breathers and continuous wave supercontinuum generation. Opt. Express, 17, 21497-21508 (2009)

28. Mussot A., Kudlinski A.: 19.5 W CW-pumped supercontinuum source from 0.65 to 1.38 $\mu \mathrm{m}$, Electron. Lett. 45, 29-30 (2009)

29. Mussot A., Kudlinski A., Kolobov M., Louvergneaux E., Douay M., Taki M.: Observation of extreme temporal events in CW-pumped supercontinuum. Opt. Express, 17, 17010-17015 (2009)

30. Solli D.R., Ropers C., Koonath P., Jalali B.: Optical rogue waves. Nat. 450, 1054 (2007)

31. Akhmediev N., Soto-Crespo J.M., Devine N.: Breather turbulence versus soliton turbulence: Rogue waves, probability density functions, and spectral features. Phys. Rev. E, 94, $022212(2016)$

32. Benjamin T.B., Feir J.E.: The disintegration of wave trains on deep water Part 1. Theory. J. Fluid Mechanics, 27, 417-430 (1967) 
33. Hasimoto H., Ono H.: Nonlinear modulation of gravity waves. J. of the Phy. Soc. of Japan, 33, 805-811 (1972)

34. Crabb M. and Akhmediev N.: Two-breather solutions for the class I infinitely extended nonlinear Schrödinger equation and their special cases. Nonlinear Dyn. 98, 245-255, (2019)

35. Akhmediev N., Korneev V.I.: Modulation instability and periodic solutions of the nonlinear Schrödinger equation. Theor. Math. Phys. 69, 1089-1093 (1986)

36. Akhmediev N., Korneev V.I., Mitskevich N.V.: N-modulation signals in a single-mode optical waveguide under nonlinear conditions. J. of Exp. and Theor. Phys. 67, 89 (1988)

37. Trillo S., Wabnitz S.: Dynamics of the nonlinear modulational instability in optical fibers. Opt. Lett. 16, 986-988 (1991)

38. Dudley J.M., Dias F., Erkintalo M., Genty G.: Instabilities, breathers and rogue waves in optics. Nat. Photonics, 8, 755-764 (2014)

39. Kedziora D.J., Ankiewicz A., Akhmediev N.: Circular rogue wave clusters. Phys. Rev. E, 84, 056611 (2011)

40. Bendahmane A., Mussot A., Szriftgiser P., Zerkak O., Genty G., Dudley J., Kudlinski A.: Experimental dynamics of Akhmediev breathers in a dispersion varying optical fiber. Opt. Lett. 39, 4490-4493 (2014)

41. Mussot A., Kudlinski A., Droques M., Szriftgiser P., Akhmediev N.: Fermi-Pasta-Ulam Recurrence in Nonlinear Fiber Optics: The Role of Reversible and Irreversible Losses. Phys. Rev. X. 4, 011054 (2014)

42. Van Simaeys G., Emplit P., Haelterman M.: Experimental demonstration of the FermiPasta-Ulam recurrence in a modulationally unstable optical wave. Phys. Rev. Lett. 87, 033902 (2001)

43. Akhmediev N., Deja vu in optics. Nat. 413, 267-268 (2001)

44. Akhmediev N., Ankiewicz A.: Solitons: nonlinear pulses and beams. Chapman \& Hall, London, (1997)

45. Akhmediev N., Eleonskii V.M., Kulagin N.E.: Exact first-order solutions of the nonlinear Schrödinger equation. Theoreticheskaya Mathematicheskaya Fizika (USSR), 72, 183 - 196. English translation in: Theor. and math. phys. 72, 809 (1987).

46. Akhmediev, N., Ankiewicz, A.: First-order exact solutions of the nonlinear Schrödinger equation in the normal-dispersion regime. Phys. Rev. A 47, 3213-3221 (1993)

47. Mihalache, D., Panoiu, N.C.: Analytic method for solving the nonlinear Schrödinger equation describing pulse propagation in dispersive optic fibres. J. Phys. A Math. Gen. 26, 2679-2697 (1993)

48. Gagnon, L.: Solitons on a continuous-wave background and collision between two dark pulses: some analytical results. J. Opt. Soc. Am. B 10, 469-474 (1993)

49. Mihalache, D., Lederer, F., Baboiu, D.M.: Two-parameter family of exact solutions of the nonlinear Schrödinger equation describing optical-soliton propagation. Phys. Rev. A 47, 3285-3290 (1993)

50. Peregrine D.H.: Water waves, nonlinear Schrödinger equations and their solutions. J. of the Australian Math. Soc. Series B. 25, 16-43 (1983)

51. Akhmediev N., Ankiewicz A., Taki M.: Waves that appear from nowhere and disappear without a trace. Phys. Lett. A, 373, 675 - 678 (2009)

52. Shrira V., Georjaev V.: What makes the Peregrine soliton so special as a prototype of freak waves?., J. of Eng. Math. 67, 11-22 (2010)

53. Akhmediev N., Soto-Crespo J.M., Ankiewicz A.: Extreme waves that appear from nowhere: On the nature of rogue waves. Phys. Lett. A, 373, 2137-2145 (2009)

54. Kedziora D.J., Ankiewicz A., Akhmediev N.: Second-order nonlinear Schrödinger equation breather solutions in the degenerate and rogue wave limits. Phys. Rev. E, 85, 066601 (2012)

55. Olver F.W.J., Lozier D.W., Boisvert R.F., Clark C.W.: NIST handbook of mathematical functions hardback and CD-ROM, Cambridge University Press, (2010)

56. Chabchoub A., Hoffmann N.P., M. O, Akhmediev N.: Super rogue waves: Observation of a higher-order breather in water waves. Phys. Rev. X. 2, 011015 (2012)

57. Coxeter, H. S. M.: Regular Polytopes, 3rd ed. New York: Dover, p. 123, (1973) 